\title{
Soft computing-based colour quantisation
}

\author{
Gerald Schaefer
}

\begin{abstract}
Soft computing techniques have shown much potential in a variety of computer vision and image analysis tasks. In this paper, an overview of recent soft computing approaches to the colour quantisation problem is presented. Colour quantisation is a common image processing technique to reduce the number of distinct colours in an image. Those selected colours form a colour palette, while the resulting image quality is directly determined by the choice of colours in the palette. The use of generic optimisation techniques such as simulated annealing and soft computing-based clustering algorithms founded on fuzzy and rough set ideas to formulate colour quantisation algorithms is discussed. These methods are capable of deriving good colour palettes and are shown to outperform standard colour quantisation techniques in terms of image quality. Furthermore, a hybrid colour quantisation algorithm which combines a generic optimisation approach with a common clustering algorithm is shown to lead to improved image quality. Finally, it is demonstrated how optimisation-based colour quantisation can be employed in conjunction with a more appropriate measure for image quality.
\end{abstract}

Keywords: Colour quantisation; Colour palette; Soft computing; Clustering; Optimisation; Image quality

\section{Introduction}

Colour quantisation is a common image processing technique that allows the representation of true colour images using only a small number of colours. True colour images typically use 24 bits per pixel resulting overall in $2^{24}$, i.e. more than 16 million, different colours. Colour quantisation uses a colour palette that contains only a small number of distinct colours (usually between 8 and 256), and pixel data are then stored as indices to this palette. Since each pixel in the image now takes on one of the colours of the palette, the choice of the colours that make up the palette is of crucial importance for the quality of the quantised image.

A common way of expressing this quality is to calculate the difference between the original (unquantised) image $O$ and its colour-quantised counterpart $Q$ for which the mean-squared error (MSE) is the most widely used measure:

$$
\begin{aligned}
\operatorname{MSE}(O, Q)= & \frac{1}{3 n m} \sum_{i=1}^{n} \sum_{j=1}^{m}\left(\left(R_{O}(i, j)-R_{Q}(i, j)\right)^{2}\right. \\
& \left.+\left(G_{O}(i, j)-G_{Q}(i, j)\right)^{2}+\left(B_{O}(i, j)-B_{Q}(i, j)\right)^{2}\right),
\end{aligned}
$$

\footnotetext{
Correspondence: gerald.schaefer@ieee.org
}

Department of Computer Science, Loughborough University, Loughborough, UK

\section{Springer}

(c) 2014 Schaefer; licensee Springer. This is an Open Access article distributed under the terms of the Creative Commons Attribution License (http://creativecommons.org/licenses/by/2.0), which permits unrestricted use, distribution, and reproduction in any medium, provided the original work is properly cited. where $R(i, j), G(i, j)$, and $B(i, j)$ are the red, green, and blue pixel values at location $(i, j)$, and $n$ and $m$ are the dimensions of images.

However, the selection of the optimal colour palette is known to be an NP-complete problem [1]. In the image processing literature, many different algorithms have been introduced that aim to find a palette that allows for good image quality of the quantised image. A relatively simple approach is the popularity algorithm [2], which typically following a uniform quantisation to 5 bits per channel - selects the $N$ colours that are represented most often to form the colour palette. In median cut quantisation [2], an iterative procedure repeatedly splits (by a plane through the median point) colour cells into sub-cells. In octree quantisation [3], the colour space is represented as an octree where sub-branches are successively merged to form the palette, while Neuquant [4] employs a onedimensional self-organising Kohonen neural network to generate the colour map.

In this paper, we present several soft computing approaches to colour quantisation. In particular, in Section 2.1, we show how general purpose optimisation algorithms such as simulated annealing can be used to derive a good colour palette. Colour quantisation can also be regarded as a clustering problem. Consequently, in Section 2.2, several soft computing-based clustering algorithms, namely fuzzy based clustering, rough set 
based clustering and a combined fuzzy-rough clustering approach, and their application to the colour quantisation problem, are discussed. In Section 2.3, we present experimental results that confirm that these soft computingbased methods do indeed make effective approaches for colour quantisation, outperforming several standard algorithms. A hybrid optimisation scheme for colour quantisation is described in Section 2.4 and shown to lead to improved performance. In Section 2.5, we show that by adapting the objective function, optimisationbased colour quantisation can be formulated, employing a more appropriate image quality metric. Finally, Section 3 concludes the paper.

\section{Review}

\subsection{Soft computing-based optimisation for colour quantisation}

The main advantage of black-box optimisation algorithms is that they do not require any domain-specific knowledge yet are able to provide a near-optimal solution. This makes them suitable for a variety of problems, and in the following, we show how they can be employed to lead to an effective colour quantisation algorithm.

While there are many different optimisation algorithms (e.g. in [5], genetic algorithms were used for colour quantisation, while in [6], particle swarm optimisation [7] was utilised), the approach in [8] employs a modification of the well-known simulated annealing algorithm. Simulated annealing (SA) was first introduced as a general optimisation method by Kirkpatrick et al. [9], and it simulates the annealing of metal, in which the metal is heated up to a temperature near its melting point and then slowly cooled down. This allows the particles to move towards a minimum energy state, with a more uniform crystalline structure. The process therefore permits some control over the microstructure.

Simulated annealing is a variation of the hill-climbing algorithm. Both start from a randomly selected point within the search space of all possible solutions. Each point in search space has a measurable error value, $E$, associated with it, which indicates the quality of the solution. From the current point in search space, new trial solutions are selected for testing from the neighborhood of the current solution. This is usually done by moving a small step in a random direction. Typically, small and equally distributed random numbers from the interval $\left[-s_{\max }, s_{\max }\right]$ are added to each component of the current solution vector, where $s_{\max }$ is called the maximum step width and is chosen from the interval between 0 and the upper limit of the search space dimension.

If the decrease in error values, denoted as $\Delta E$, is negative (i.e. the error of a trial solution is below that of the current one), then the trial solution is accepted as the current solution. However unlike hill-climbing, SA does not automatically reject a new candidate solution if $\Delta E$ is positive. Instead, it becomes the current solution with probability $p(T)$ which is usually determined using

$$
p(T)=e^{-\Delta E / T},
$$

where $T$ is referred to as 'temperature', an abstract control parameter for the cooling schedule. For a given temperature and positive values of $\Delta E$, the probability function shown in Equation 2 has a defined upper limit of 1 and tends towards 0 for large positive values of $\Delta E$.

The algorithm starts with a high temperature, i.e. with a high transition probability. The temperature is then reduced towards 0 , usually in steps, according to a cooling schedule such as

$$
T_{n+1}=\alpha T_{n},
$$

where $T_{n}$ is the temperature at step $n$ and $\alpha$ is a cooling coefficient (usually between 0.8 and 0.99 ).

During each step, the temperature must be held constant for an appropriate number of iterations in order to allow the algorithm to settle into a 'thermal equilibrium', i.e. a balanced state. If the number of iterations is too small, the algorithm is likely to converge to a local minimum.

For both continuous parameter optimisation and discrete parameters with large search ranges, it is practically impossible to choose direct neighbours of the current solution as new candidate solutions due to the vast number of points in the search space. Therefore, it is necessary to choose new candidates at some distance in a random direction of the current solution in order to navigate through the search space in an acceptable time. This distance could either be a fixed step width $s$ or it could have an upper limit, $s_{\max }$. The maximum step width $s_{\max }$ is crucial to the success of SA. If $s_{\max }$ is too small and the start point for a search run is too far away from the global optimum, the algorithm might not be able to get near that optimum. If, on the other hand, the step width is too large and the peak of the optimum very narrow, the algorithm might never reach the top because most of the steps are too large.

Step width adapting simulated annealing (SWASA) [10] overcomes the problems associated with constant values for $s_{\max }$ using a scaling function to adapt the maximum step width to the current iteration by

$$
s_{\max }(n)=\frac{2 s_{0}}{1+e^{\beta n / n_{\max }}},
$$

where $s_{\max }(n)$ is the maximum step width at iteration $n$, $s_{0}$ is the initial maximum step width, $n_{\max }$ the maximum number of iterations, and $\beta$ is an adaptation constant.

For colour quantisation, the objective is, as mentioned, to minimise the total error introduced through the application of a colour palette. The colour palette $C$ for an 
image $I$, a codebook of $k$ colour vectors, should then be chosen so as to minimise the error function

$$
E(C, I)=\frac{1}{\sum_{j=1}^{k} l_{j}} \sum_{i=1}^{k} \sum_{j=1}^{l_{i}}\left\|C_{i}-I_{j}\right\|+p(C, I),
$$

with

$$
p(C, I)=\sum_{i=1}^{k} \delta a_{i}, \quad a_{i}= \begin{cases}1 & \text { if } l_{i}=0 \\ 0 & \text { otherwise }\end{cases}
$$

where $l_{i}$ is the number of pixels $I_{j}$ represented by colour $C_{i}$ of the palette, $\|$.$\| is the Euclidean distance in RGB (red-$ green-blue) space, and $\delta$ is a constant (set to $\delta=10$ in [8]). The objective function $E(C, I)$ used is hence a combination of the mean Euclidean distance, i.e. the error measure of Equation 1, and a penalty function. The purpose of the penalty function $p(C, I)$ is to avoid unused palette colours by adding a constant penalty value to the error for each entry in the codebook that is not used in the resulting picture.

\subsection{Soft computing-based clustering for colour quantisation}

Colour quantisation can also be seen as a clustering problem where the task is to identify those clusters that best represent the colours in an image. Consequently, soft computing-based clustering algorithms can be readily adapted to derive a good colour palette. In the following, we discuss how fuzzy c-means, rough c-means, and a combined fuzzy-rough clustering algorithm can be employed for colour quantisation.

\subsubsection{Fuzzy c-means}

Fuzzy c-means (FCM) is based on the idea of finding cluster centres by iteratively adjusting their positions and evaluation of an objective function as in (hard) c-means, yet allows more flexibility by introducing the possibility of partial memberships to clusters. The general FCM algorithm is illustrated in Figure 1.
For colour quantisation, the error function follows the form

$$
E=\sum_{j=1}^{C} \sum_{i=1}^{N} \mu_{i j}^{k}\left\|x_{i}-c_{j}\right\|^{2},
$$

where $\mu_{i j}^{k}$ is the fuzzy membership of pixel $x_{i}$ and the colour cluster identified by its centre $c_{j}$, and $k$ is a constant that defines the fuzziness of the resulting partitions.

$E$ can reach the global minimum when pixels nearby the centroid of corresponding clusters are assigned higher membership values, while lower membership values are assigned to pixels far from the centroid [11]. Here, the membership is proportional to the probability that a pixel belongs to a specific cluster where the probability is only dependent on the distance between the image pixel and each independent cluster centre. The membership functions and the cluster centres are updated by

$$
\mu_{i j}=\frac{1}{\sum_{m=1}^{C}\left(\frac{\left\|x_{j}-c_{i}\right\|}{\left.\left\|x_{j}-c_{m}\right\|\right)^{2 /(k-1)}}\right)},
$$

and

$$
c_{i}=\frac{\sum_{j=1}^{N} \mu_{i j}^{k} x_{j}}{\sum_{j=1}^{N} \mu_{i j}^{k}} .
$$

Fuzzy c-means operates iteratively though the following steps [12]:

Step 1. Initialise the cluster centres $c_{i}$ and let $t=0$.

Step 2. Initialise the fuzzy partition memberships functions $\mu_{i j}$ according to Equation 8.

Step 3. Let $t=t+1$ and compute new cluster centres $c_{i}$ using Equation 9.

Step 4. Repeat steps 2 to 3 until convergence.

An initial setting for each cluster centre is required, and FCM is guaranteed to converge to a local minimisation solution. The efficiency of FCM has been comprehensively investigated in [13]. To address the inefficiency of the original FCM algorithm, several variants of the fuzzy c-means algorithm have been introduced which are discussed in the following.
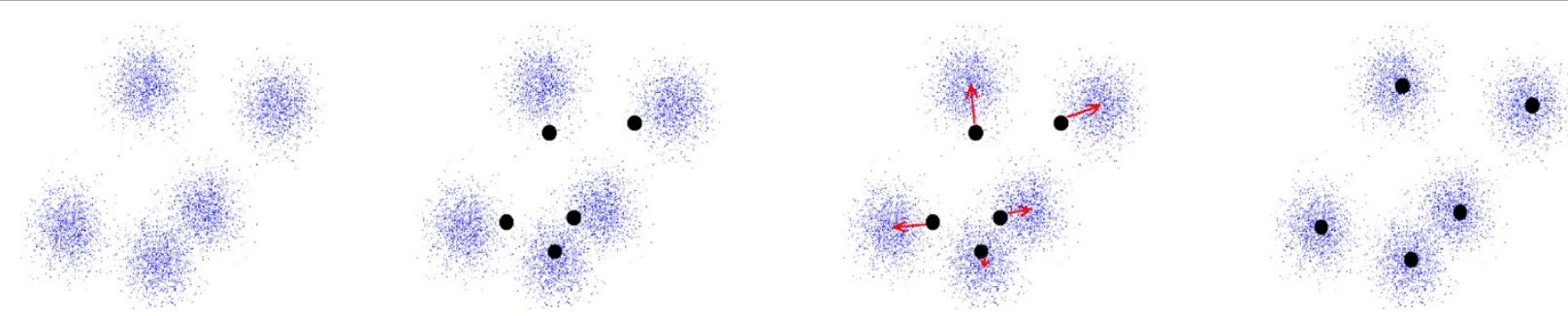

Figure 1 Illustration of FCM, from left to right: data to clusters - random cluster centres - clusters converging - final settlement. 


\subsubsection{Fuzzy c-means variants}

While FCM often provides good clustering results, it also suffers from a relatively high computational complexity, especially when there are many samples as is the case for colour quantisation. However, a number of faster FCM variants have been developed and have also been shown to work well for colour quantisation [14].

To combat the computational complexity of FCM, Cheng et al. [15] proposed a multistage random sampling strategy. This method has a lower number of feature vectors and also needs fewer iterations to converge. The basic idea is to randomly sample and obtain a small subset of the dataset in order to approximate the cluster centres of the full dataset. This approximation is then used to reduce the number of iterations. Random sampling FCM (RSFCM) consists of two phases. First, a multistage iterative process of a modified FCM is performed. In the second phase, standard FCM is performed with the cluster centres approximated by the final cluster centres from the first phase. It has been shown that RSFCM is able to reduce the computational complexity compared to the classical FCM method.

Ahmed et al. [16] introduced an alternative to classical FCM by adding a term that enables the labelling of a pixel to be associated with its neighbourhood. As a regulator, the neighbourhood term can change the solution towards piecewise homogeneous labelling. As a further extension, in [17], the enhanced FCM (EnFCM) algorithm was presented. In order to reduce the computational complexity, a linearity-weighted sum image is formed from the original image, and a modified objective function is employed. EnFCM considers a number of pixels with similar colours as a weight. Thus, this approach can accelerate the convergence of searching for global similarity.

Anisotropic mean shift-based FCM (AMSFCM) is an efficient approach to fuzzy c-means clustering which utilises an anisotropic mean shift algorithm coupled with fuzzy clustering [14]. Mean shift-based techniques have been shown to be capable of estimating the local density gradients of similar pixels. These gradient estimates are iteratively performed so that all pixels can find similar pixels in the same image [18]. AMSFCM combines fuzzy c-means and anisotropic mean shift, a mean shift variant that does not suffer of shortcomings due to radially symmetric kernels [19]. Importantly, the AMSFCM algorithm continuously inherits and updates the states, based on the mutual correction of FCM and mean shift.

\subsubsection{Rough c-means}

Lingras and West [20] introduced a rough set-inspired clustering algorithm based on the well-known c-means algorithm. In this rough c-means (RCM) approach, each cluster $c_{k}$ is described not only by its centre $m_{k}$, but also contains additional information, in particular its lower approximation $c_{k}$, its upper approximation $\overline{c_{k}}$, and its boundary area $\overline{c_{k}^{b}}=\overline{c_{k}}-\underline{c_{k}}$. The clustering algorithm proceeds in the following steps:

Step 1. Each data sample is randomly assigned to one lower approximation. Since the lower approximation of a cluster is a subset of its upper approximation, this also automatically assigns the sample to the upper approximation of the same cluster.

Step 2. The cluster centres are updated as

$$
m_{k}=\left\{\begin{array}{l}
\omega_{l} \sum_{x_{i} \in \underline{c_{k}}} \frac{x_{i}}{\mid \underline{c_{k} \mid}}+\omega_{b} \sum_{x_{i} \in c_{k}^{b}} \frac{x_{i}}{\left|c_{k}^{b}\right|} \text { if } c_{k}^{b} \neq\{\} \\
\omega_{l} \sum_{x_{i} \in \underline{c_{k}}} \frac{x_{i}}{\left|\underline{c_{k}}\right|} \text { otherwise }
\end{array} .\right.
$$

The cluster centres are hence determined as a weighted average of the samples belonging to the lower approximation and the boundary area, where the weights $\omega_{l}$ and $\omega_{b}$ define the relative importance of the two sets.

Step 3. For each data sample, the closest cluster centre is determined and the sample is assigned to its upper approximation. Then, all clusters that are at most $\epsilon$ further away than the closest cluster are determined. If such clusters exist, the sample will also be assigned to their upper approximations. If no such cluster exists, the sample is assigned also to the lower approximation of the closest cluster.

Step 4. If the algorithm has converged (i.e. if the cluster centres do not change any more, or after a pre-set number of iterations), terminate; otherwise, go to step 2 .

Strictly speaking, this algorithm does not implement all properties set out for rough sets [21] and hence belongs to the reduced interpretation of rough sets as lower and upper approximations of data [22].

Peters [23] pointed out some potential pitfalls of the algorithm in terms of objective function and numerical stability and suggested some improvements to overcome these. Equation 10 is revised to

$$
m_{k}=\omega_{l} \sum_{x_{i} \in \underline{c_{k}}} \frac{x_{i}}{\left|\underline{c_{k}}\right|}+\omega_{u} \sum_{x_{i} \in \overline{c_{k}}} \frac{x_{i}}{\left|\overline{c_{k}}\right|}
$$

with $\omega_{l}+\omega_{u}=1$, i.e. as a convex combination of lower and upper approximation means. In order to overcome 
the possibility of situations with empty lower approximations, the calculation of cluster centres can be modified so that for empty lower approximations, the cluster centre is calculated as the average of samples in the upper approximation or by ensuring that each lower approximation has at least one member.

In [24], an RCM-based colour quantisation is introduced which follows the clustering approach from [20] and [23], assigning the data sample closest to the cluster centre to its lower approximation, though with a different initialisation approach where rather than randomly assigning samples to clusters, random cluster centres are generated first and then the algorithm proceeds with steps 3 , 2, and 4 (i.e., steps 2 and 3 reversed) in order to derive a colour palette.

\subsubsection{Fuzzy-rough c-means}

Fuzzy-rough c-means (FRCM) clustering [25] utilises, in addition to the fuzziness of fuzzy c-means, concepts of rough set theory to provide an effective clustering algorithm that can also be adapted for colour quantisation [26]. In particular, as in rough c-means, each cluster is represented by a lower and an upper approximation. However, while the lower approximation is defined as crisp, the boundary area is fuzzy. While the aim is to minimise the same error function $E$ as in Equation 7, memberships are defined as

$$
\mu_{i j}=\left\{\begin{array}{cc}
1 & \forall x_{i} \in \underline{c_{j}} \\
\frac{1}{\sum_{l=1}^{C}\left(\frac{\left\|x_{i}-m_{j}\right\|}{\left.\left\|x_{i}-m_{l}\right\|\right)^{2 /(\alpha-1)}}\right)} \forall x_{i} \in c_{j}^{b} .
\end{array}\right.
$$

Calculation of cluster centres $m_{j}$ remains as given in Equation 9, while lower and upper approximations are defined as detailed in Section 2.2.3 for rough c-means. The difference between fuzzy c-means and fuzzy-rough cmeans is hence that the membership values in the lower approximation are 1 (i.e. crisp), while those in the boundary region are fuzzy memberships. In other words, fuzzyrough c-means first partitions the data into two classes: lower approximation and boundary area, and only those points in the boundary region are fuzzified. This in turn leads to faster convergence when compared to FCM [25]. In contrast to rough c-means, samples in the boundary region are not all treated equally but are rather assigned membership values depending on their distance to the cluster centroids. In addition, there is no need to specify weights for the calculation for cluster centroids.

\subsection{Colour quantisation performance}

In order to evaluate the various colour quantisation algorithms, we performed a set of experiments on a set of six test images. These images, Lenna, Peppers, Mandrill,
Sailboat, Airplane, and Pool, are commonly used in the colour quantisation literature and hence present a good test bed for evaluation purposes. We applied all seven discussed algorithms, that is SWASA, FCM, RSFCM, EnFCM, AMSFCM, RCM, and FRCM, to all images to generate quantised images with a palette of 16 colours.

For the simulated annealing algorithm, a populationbased version of the SWASA algorithm with a population size of 10 was employed. The start temperature $T_{0}$ was chosen to be 20, and the cooling coefficient $\alpha$ was set to 0.9 . The parameters $s_{0}$ and $\beta$ were set to 100 and 5.3 , respectively. The temperature was kept constant for over 20 iterations, and the maximum number of iterations was set to 10,000 . For the rough c-means approach, we adopted the parameters $\omega_{l}=0.7, \omega_{u}=0.3$, and $\tau=$ 0.001 (image pixel values are normalised to $[0 ; 3]^{3}$ ). For the fuzzy c-means and fuzzy rough c-means algorithms, the fuzziness exponent $\alpha$ was set to 1.2.

To put the results we obtained into context, we also implemented four popular colour quantisation algorithms (which are often integrated in typical image processing software) to generate corresponding quantised images with palette size 16 . The algorithms we tested were as follows:

- Popularity algorithm [2]: Following a uniform quantisation to 5 bits per channel, the $N$ colours that are represented most often form the colour palette.

- Median cut quantisation [2]: An iterative algorithm that repeatedly splits (by a plane through the median point) colour cells into sub-cells.

- Octree quantisation [3]: The colour space is represented as an octree where sub-branches are successively merged to form the palette.

- Neuquant [4]: A one-dimensional self-organising Kohonen neural network is applied to generate the colour map.

For all algorithms, pixels in the quantised images were assigned to their nearest neighbours in the colour palette to provide the best possible image quality.

The obtained results are listed in Table 1, expressed in terms of average (over 10 runs of the algorithms) peaksignal-to-noise ratio (PSNR) defined as

$$
\operatorname{PSNR}(O, Q)=10 \log _{10} \frac{255^{2}}{\operatorname{MSE}(O, Q)}
$$

with $\operatorname{MSE}(O, Q)$ calculated as in Equation 1. A higher PSNR hence indicates better image quality.

As can be seen from Table 1, all the soft computing algorithms provide very good results and clearly outperform standard colour quantisation algorithms. The results 
Table 1 Quantisation results, given in terms of PSNR (dB) with the best result for each image in boldface

\begin{tabular}{|c|c|c|c|c|c|c|c|}
\hline & Lenna & Peppers & Mandrill & Sailboat & Pool & Airplane & average \\
\hline Popularity algorithm [2] & 22.24 & 18.56 & 18.00 & 8.73 & 19.87 & 15.91 & 17.22 \\
\hline Median cut [2] & 23.79 & 24.10 & 21.52 & 22.01 & 24.57 & 24.32 & 23.39 \\
\hline Octree [3] & 27.45 & 25.80 & 24.21 & 26.04 & 29.39 & 28.77 & 26.94 \\
\hline Neuquant [4] & 27.82 & 26.04 & 24.59 & 26.81 & 27.08 & 28.24 & 26.73 \\
\hline SWASA [8] & 27.79 & 26.16 & 24.46 & 26.69 & 29.84 & 29.43 & 27.40 \\
\hline FCM [14] & 28.81 & 26.77 & 25.03 & 27.25 & 31.03 & 30.23 & 28.17 \\
\hline RSFCM [14] & 28.70 & 26.70 & 24.98 & 27.32 & 30.81 & 30.73 & 28.20 \\
\hline EnFCM [14] & 28.61 & 26.74 & 24.87 & 27.22 & 31.11 & 29.92 & 28.08 \\
\hline AMSFCM [14] & 28.63 & 26.71 & 24.66 & 27.24 & 30.87 & 29.96 & 28.01 \\
\hline RCM [24] & 28.63 & 26.67 & 25.02 & 27.62 & 29.40 & 30.50 & 27.98 \\
\hline FRCM [26] & 28.44 & 26.80 & 25.03 & 27.47 & 31.20 & 31.24 & 28.73 \\
\hline
\end{tabular}

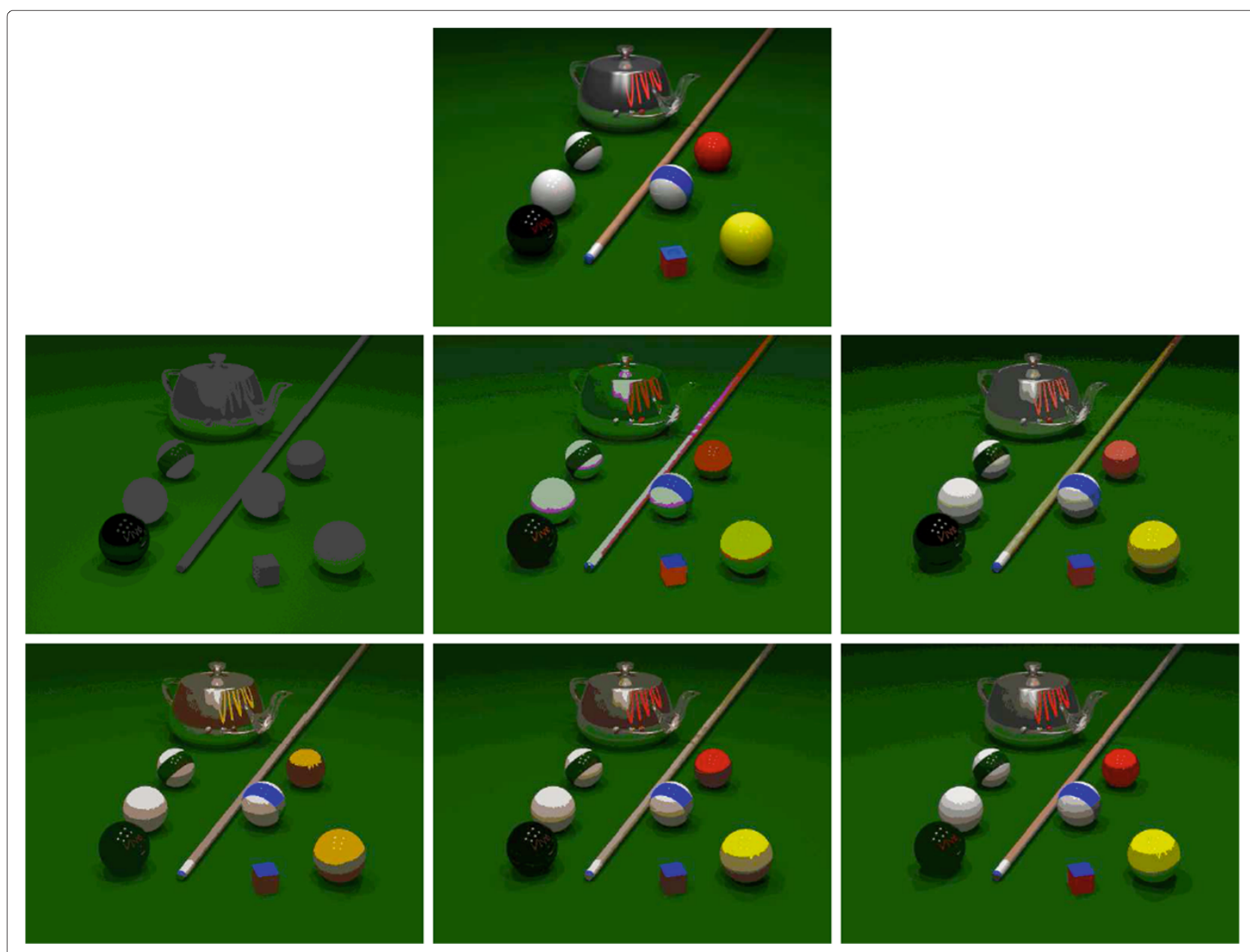

Figure 2 Results of colour quantisation algorithms applied to the Pool image (top row). Shown are the quantised images after applying (from left to right, top to bottom) popularity, median cut, octree, Neuquant, rough c-means, and fuzzy-rough c-means algorithms. 
of the different fuzzy clustering approaches are fairly similar which suggests that the computationally more efficient versions (RSFCM, EnFCM, AMSFCM) can be employed without sacrificing image quality. Also, the rough set approach gives similar performance and the presented rough colour quantisation approach hence adds to the applications of rough sets in the field of imaging and vision. The best performance is achieved by the combined fuzzy-rough c-means approach, which gives the best image quality for four of the six images and overall provides a PSNR improvement of more than 0.5 compared to the next ranked algorithm.

Figure 2 shows the Pool image together with the images colour quantised by the popularity, median cut, octree, and Neuquant algorithms as well as two of the softcomputing based techniques, namely the rough c-means and fuzzy-rough c-means approaches. It is clear that the popularity algorithm performs poorly on this image and assigns virtually all of the colours in the palette to green and achromatic colours. Median cut is better but still provides a fairly poor colour reproduction; most of the colours in the quantised image are fairly different from the original. The same holds true for the images produced by Neuquant. Here, the most obvious artefact is the absence of an appropriate red colour in the colour palette. A far better result is achieved by the octree algorithm, although here also, the red is not very accurate and the colour of the cue is greenish instead of brown. Clearly better image quality is maintained by applying the rough c-means algorithm. Although the colour palette has only 16 entries, all colours of the original image are accurately presented including the red ball and the colour of the billiard cue. A further improvement is achieved by the fuzzy-rough cmeans colour quantisation technique which achieves even better colour reproduction, e.g. in the reflections on the black ball.

\subsection{Hybrid optimisation-based colour quantisation}

Figure 3 shows a typical run of the simulated annealing optimisation method applied to colour quantisation as explained in Section 2.1. The solid line represents the average quantisation error over time (iterations) while the dashed line represents the best solution of each iteration.

As can be seen in Figure 3, there is always a variation in error values within the population which indicates that although simulated annealing is able to find good solutions (as was confirmed in Section 2.3), i.e. solutions from within the region around the global optimum, it rarely exploits that region completely. Therefore, in [27], SWASA was combined with a standard c-means clustering algorithm [28] to provide a stacked hybrid optimisation method. C-means clustering is guaranteed to converge towards the local clustering minimum by iteratively carrying out the following two steps:

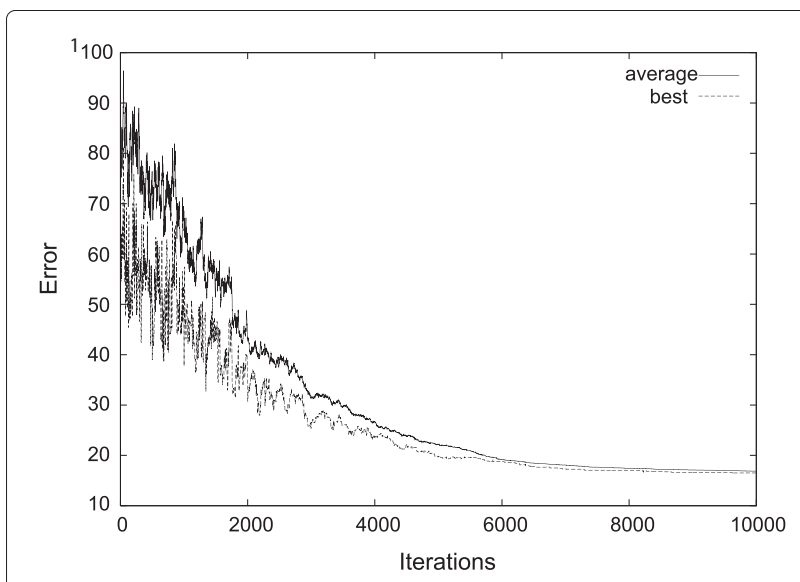

Figure 3 Typical run of SA optimisation for colour quantisation [27].

- Each input vector is mapped to its closest codeword by a nearest neighbour search.

- The input vectors assigned in each class (i.e. for each codeword) are represented by the centroid of the vectors in that class.

In this hybridised algorithm, the SA component is hence responsible for identifying the region in the search space that will contain the global optimum while the c-means component will then descend into the minimum present in that region.

To show the effect of this hybridisation, we ran the combined algorithm on the same image dataset that was used in Section 2.3, and show the results, again in terms of PSNR, in Table 2. As can be seen from there, the further adjustment through application of a subsequent clustering step does indeed improve image quality significantly, resulting in a colour quantisation algorithm that also outperforms all algorithms from Table 1.

\subsection{Image quality metric-based colour quantisation}

Although many colour quantisation algorithms have been proposed, virtually all of them define the goal of quantisation as that of finding a palette that minimises the MSE, given in Equation 1, of the resulting image. It is however well known that metrics such as MSE and PSNR do not correspond very well to how the human visual system operates and how humans judge differences between images. An image with a higher MSE does not necessarily have perceptually lower image quality; similarly, two images that are perceived with equal quality are likely to have different values in terms of MSE or PSNR.

Furthermore, RGB, the device space, is poorly related to the human visual system. Colour differences calculated as Euclidean distances between RGB co-ordinates do not 
Table 2 Quantisation results, given in terms of PSNR (dB), of the hybrid SA algorithm

\begin{tabular}{lccccccc}
\hline & Lenna & Peppers & Mandrill & Sailboat & Pool & Airplane & average \\
\hline SWASA [8] & 27.79 & 26.16 & 24.46 & 26.69 & 29.84 & 29.43 & 27.40 \\
Hybrid SWASA [27] & 29.70 & 27.17 & 25.37 & 27.95 & 31.57 & 32.94 & 28.97 \\
\hline
\end{tabular}

correspond well to how humans perceive colour differences. Much research has been done on deriving colour spaces such as CIELAB and CIELUV that are designed to be perceptually uniform, i.e. where Euclidean distances correspond to perceived distances in all regions of the colour space [29]. Uniform colour spaces have been used in colour quantisation algorithms and have been shown to perform better compared to algorithms based on the RGB space [30].

However, the application of a perceptual uniform colour space alone does not guarantee improved image quality. Uniform colour spaces were developed for and hence only accurately model the colour differences between large patches of uniformly coloured samples. Real images on the other hand seldomly comprise such large areas. Therefore, it is crucial to also take into account the spatial characteristics of images when developing an appropriate image quality metric [31].

S-CIELAB (for spatial CIELAB [32]) first converts the RGB image into an opponent colour space. The individual channels are then convolved with a kernel whose shape is estimated from the visual spatial sensitivity to that channel. This convolution simulates the blurring that occurs in the human visual system. Both the opponent colour space and the convolution kernels were derived following a series of experiments on the pattern-colour separability of the visual system [33]. After the filtering, the image is converted to XYZ and then to CIELAB to provide spatial CIELAB co-ordinates. The difference between two images can then be expressed as the average colour difference, expressed in terms of $\Delta E$ units, between the two $S$-CIELAB representation, where the colour difference is usually calculated as the Euclidean distance between two colours.

In contrast to colour spaces such as CIELAB, for which colour quantisation can be easily adapted [30] by converting the RGB image to the new colour space and then applying the quantisation algorithm, this is not possible for S-CIELAB. The reason is that S-CIELAB does not simply provide a new colour space but takes into account the spatial interaction between neighbouring pixels. It is therefore image dependent, and identical S-CIELAB coordinates in two different images can originate from fairly different original RGB values. In the context of colour quantisation, this means that converting an image to $\mathrm{S}$ CIELAB and performing the quantisation there will not lead to an optimal palette. Rather, the palette has to be found in the palette search space, and then the image quality is calculated based on the quantised image. This is possible using optimisation techniques such as the SWASA algorithm explained in Section 2.1 to develop an image metric-based colour quantisation algorithm [34]. The objective function to be minimised here is defined as the average $\Delta E$ between the original image $O$ and the image quantised using the colour palette.

That this leads indeed to improved performance is again evaluated on the same image dataset as in Section 2.3. The results are given in Table 3. From there, it is evident that optimisation-based colour quantisation based on minimising MSE clearly outperforms conventional algorithms also when expressed in terms of S-CIELAB image quality. However, directly optimising with respect to this image metric leads to a significant further improvement, reducing the average $\Delta E$ from 6.65 to 4.37 .

\section{Conclusions}

In this paper, we have given an overview of recent soft computing-based colour quantisation approaches and have shown that this family of algorithms work very well, resulting in quantised images with high image quality. In particular, we have discussed the use of optimisation algorithms such as simulated annealing and of soft computing-based clustering algorithms including fuzzy $\mathrm{c}$-means, rough c-means, and combined fuzzy-rough

Table 3 Quantisation results, given in terms of $\Delta E_{S-C I E L A B}$

\begin{tabular}{|c|c|c|c|c|c|c|c|}
\hline & Lenna & Peppers & Mandrill & Sailboat & Pool & Airplane & average \\
\hline Popularity algorithm [2] & 11.92 & 21.81 & 20.54 & 41.03 & 9.31 & 15.59 & 20.03 \\
\hline Median cut [2] & 10.34 & 8.89 & 10.66 & 10.10 & 7.73 & 7.05 & 9.13 \\
\hline Octree [3] & 7.20 & 7.92 & 9.36 & 6.66 & 4.85 & 3.57 & 6.59 \\
\hline Neuquant [4] & 7.13 & 7.84 & 9.79 & 5.50 & 5.65 & 4.21 & 6.68 \\
\hline SWASA/MSE [8] & 6.78 & 7.77 & 9.28 & 6.48 & 5.69 & 3.87 & 6.65 \\
\hline SWASA/S-CIELAB [34] & 4.57 & 6.33 & 6.27 & 3.50 & 3.06 & 2.47 & 4.37 \\
\hline
\end{tabular}


c-means approaches in this context. All techniques were compared against standard colour quantisation methods and were shown to clearly outperform them. A hybrid colour quantisation algorithm, combining simulated annealing with a c-means clustering algorithm, was shown to lead to improved performance, while by modifying the objective function, optimisation-based colour quantisation algorithms can be tuned with respect to a particular image quality metric. Although the compiled results convincingly demonstrate that soft computingbased methods are well suited for the colour quantisation problem, it should also be noted that they typically have higher demands in terms of computational complexity and might hence be suitable only for situations where the quantisation stage is not time critical.

\section{Competing interests}

The author declares that he has no competing interests.

Received: 11 April 2012 Accepted: 9 January 2014

Published: 28 January 2014

\section{References}

1. XWu, Color quantization by dynamic programming and principal analysis. ACM Trans. Graph. 11(4), 348-372 (1992)

2. P.S Heckbert, Color image quantization for frame buffer display. ACM Comput. Graph. (ACM SIGGRAPH '82 Proc.) 16(3), 297-307 (1982)

3. M Gervautz, W Purgathofer, A simple method for color quantization: octree quantization, in Graphics Gems, ed. by A. S. Glassner (ACM, San Diego, 1990), pp. 287-293

4. A. H Dekker, Kohonen neural networks for optimal colour quantization. Network: Comput. Neural Syst. 5, 351-367 (1994)

5. P Scheunders, A genetic c-means clustering algorithm applied to color image quantization. Pattern Recognit. 30(6), 859-866 (1997)

6. M Omran, A Engelbrecht, A Salman, A color image quantization algorithm based on particle swarm optimization. Informatica. 29, 263-271 (2005)

7. J Kennedy, R Eberhart, Particle swarm optimization. IEEE Int. Conference on Neural Networks. 4, 1942-1948 (1995)

8. G Schaefer, L Nolle, An optimisation approach to colour palette generation. Int. J. Pattern Recognit. Mach. Intell. 1(1), 40-46 (2006)

9. S Kirkpatrick, CD Gelatt, MP Vecchi, Optimization by simulated annealing. Science. 220(4598), 671-680 (1983)

10. L Nolle, On the effect of step width selection schemes on the performance of stochastic local search strategies, in 18th European Simulation Multi-Conference, ESM 2004, Magdeburg (Gruner, Druck, Erlangen, 2004), pp. 149-153

11. KChuang, S Tzeng, H Chen, J Wu, T Chen, Fuzzy c-means clustering with spatial information for image segmentation. Comput. Med. Imaging Graph. 30, 9-15 (2006)

12. J Bezdek, A convergence theorem for the fuzzy isodata clustering algorithms. IEEE Trans. Pattern Anal. Mach. Intell. 2, 1-8 (1980)

13. R Hu, L Hathaway, On efficiency of optimization in fuzzy c-means. Neural, Parallel Sci. Comput. 10, 141-156 (2002)

14. G Schaefer, $\mathrm{H}$ Zhou, Fuzzy clustering for colour reduction in images. Telecommun. Syst. 40(1-2), 17-25 (2009). doi:10.1007/s11235-008-9143-8

15. TCheng, D Goldgof, L Hall, Fast fuzzy clustering. Fuzzy Sets Syst. 93, 49-56 (1998)

16. M Ahmed, S Yamany, Mohamed N, A Farag, T Moriaty, A modified fuzzy c-means algorithm for bias field estimation and segmentation of MRI data. IEEE Trans. Med. Imaging. 21, 193-199 (2002)

17. L Szilagyi, Z Benyo, SM Szilagyii, HS Adam, MR brain image segmentation using an enhanced fuzzy c-means algorithm, in 25th IEEE International Conference on Engineering in Medicine and Biology, vol. 1 (IEEE, Piscataway, 2003), pp. 724-726

18. D Comaniciu, P Meer, Mean shift: a robust approach toward feature space analysis. IEEE Trans. Pattern Anal. Mach. Intell. 24, 603-619 (2002)
19. J Wang, B Thiesson, Y Xu, M Cohen, Image and video segmentation by anisotropic kernel mean shift, in 8th European Conference on Computer Vision (Springer, Berlin, 2004), pp. 238-2492

20. $P$ Lingras, $C$ West, Interval set clustering of web users with rough k-means. J. Intell. Inform. Syst. 23, 5-16 (2004)

21. Z Pawlak, Rough sets. Int. J. Inform. Comput. Sci. 11, 145-172 (1982)

22. YY Yao, X Li, TY Lin, Q Liu, Representation and classification of rough set models, in Soft Computing: Third International Workshop on Rough Sets and Soft Computing: (RSSC94), ed. by TY Lin, A Martin Wildberger (Society for Computer Simulation International, San Diego, 1994), pp. 630-637

23. G Peters, Some refinements of rough k-means clustering. Pattern Recognit. 39, 1481-1491 (2006)

24. G Schaefer, H Zhou, M Celebi, A Hassanien, Rough colour quantisation. Int. J. Hybrid Inform. Syst. 8(1), 25-30 (2011). doi10.3233/HIS-2011-0128

25. Q Hu, D Yu, An improved clustering algorithm for information granulation, in Fuzzy Systems and Knowledge Discovery, ed. by L Wang, Y Jin (Springer, Heidelberg, 2005), pp. 494-504

26. G Schaefer, G Hu, H Zhou, J Peters, A Hassanien, Rough c-means and fuzzy rough c-means for colour quantisation. Fundam. Inform. 119(1), 113-120 (2012). doi10.3233/FI-2012-729

27. L Nolle, G Schaefer, Color map design through optimization. Eng. Optimization. 39(3), 327-343 (2007)

28. Y Linde, A Buzo, RM Gray, An algorithm for vector quantizer design. IEEE Trans. Commun. 28, 84-95 (1980)

29. B Hill, T Roger, FW Vorhagen, Comparative-analysis of the quantization of color spaces on the basis of the CIELAB color-difference formula. ACM Trans. Graph. 16(2), 109-154 (1997)

30. RS Gentile, JP Allebach, E Walowit, Quantization of color images based on uniform color spaces. J. Imaging Technol. 16, 11-21 (1990)

31. MP Eckert, AP Bradley, Perceptual quality metrics applied to still image compression. Signal Process. 70, 177-200 (1998)

32. X Zhang, BA Wandell, A spatial extension of CIELAB for digital color image reproduction. Journal of the Society for Information Display. 5(1), 61-63 (1997)

33. AB Poirson, BA Wandell, Pattern-colour separable pathways predict sensitivity to simple coloured patterns. Vis. Res. 36(4), 515-526 (1996)

34. G Schaefer, L Nolle, Quality metric based colour palette optimisation, in 2006 IEEE International Conference on Image Processing (IEEE, Piscataway, 2006), pp. 1793-1796

doi:10.1186/1687-5281-2014-8

Cite this article as: Schaefer: Soft computing-based colour quantisation. EURASIP Journal on Image and Video Processing 2014 2014:8.

\section{Submit your manuscript to a SpringerOpen ${ }^{\circ}$ journal and benefit from:}

- Convenient online submission

- Rigorous peer review

- Immediate publication on acceptance

- Open access: articles freely available online

- High visibility within the field

- Retaining the copyright to your article

Submit your next manuscript at $>$ springeropen.com 